\title{
Investigation of the proton beam entry window cooling of liquid metal target of a spallation neutron source using infrared thermography
}

\author{
J.A. Patorski *a , F. Groeschel ${ }^{\text {a }}$ \\ ${ }^{a}$ Spallation Neutron Source Div., Paul Scherrer Institute, CH-5232 Villigen-PSI, Switzerland
}

\begin{abstract}
During the MEGAPIE Integral Test (MIT) of the 1MW liquid metal (LM) target for a spallation neutron source, infrared thermography (IRT) has been used to investigate the liquid lead-bismuth eutectic (LBE) cooling of the proton beam entry window of the target. The IRT investigations have been performed on the real MEGAPIE target on the MIT stand (MITS) at Paul Scherrer Institute (PSI) in Switzerland, few months before of the proton irradiation period of the target. In meantime the target has been successfully irradiated (from August to December 2006) at the PSI Spallation Neutron Source (SINQ) facility; for more details see web page http://megapie.web.psi.ch/.

The Goals of the MIT thermo-hydraulics investigations of the liquid LBE "flow in flow" window cooling configuration (ca. $1.25 \mathrm{~m} / \mathrm{s}$ speedy by-pass jet flow into the ca. $0.33 \mathrm{~m} / \mathrm{s}$ main flow), have bifocal perspective.

- On one hand the goal was visualization for an observation of changes of the target cooling field pattern of the by-pass jet flow, i.e. the qualitative investigation of cooling behavior in terms of the sufficient geometrical covering of the proton beam irradiation footprint area.

- On second hand the goal was determination of local convection heat transfer coefficient (HTC) on the steel wall of the proton beam entry window area of the MEGAPIE target, i.e. the quantitative values and distribution of a cooling.

For the qualitative visualization of the real, "in situ", target window cooling we have take advantage of slightly higher temperature of the by-pass jet flow LBE streaming (ca. $4^{\circ} \mathrm{K}$ ) and we have performed so called "MIT-Warm-Jet" experiment series. For the quantitative determination of HTC instead of the real target window we have used specimen sensor dish and performed so called "MIT-KILOPIE" experiment series using the two dimensional and dynamic infrared thermography (2DD-IRT) method, which has been developed and tested in 2005 at PSI; reported during Thermosense XVIII in 2006 [1].

The results of measurements are presented in form of IRT thermograms or thermogram sequences which are extracted from the raw temperature field measurements. The in December 2006 successfully finished irradiation period of MEGAPIE project and the integrity of the whole target have shown and proved the predicted sufficiency of the window cooling.
\end{abstract}

Keywords: quantitative IR thermography, thermo-hydraulics, liquid metal coolant, local convection heat transfer coefficient, MEGAPIE, spallation neutron source, liquid metal target

\section{INTRODUCTION}

A new concept of a high power neutron spallation source with a liquid metal (LM) target is developed within the international MEGAPIE Project [2]. The $600 \mathrm{MeV}$ proton beam penetrates into the target through a beam entry window.

This window will be strongly heated; the heat deposition in the steel wall will produce a heat flux $q^{*}$ of up to 140 $\mathrm{W} / \mathrm{cm}^{2}$. The LM, in this case lead-bismuth eutectic (LBE) is simultaneously used as target material and coolant. It is contained in approx. $4 \mathrm{~m}$ long structure of concentric pipes and vessels (see Fig.1a). The LBE flow-guiding part of the target consists of two steel pipes placed in the middle of the cross section of the target. The lower part of the outer pipe is closed with a hemispherical shell, forming a vertical, cylindrical LM container and causing a "U-form" flow i.e. $180^{\circ}$-change of a downward LBE main (MP) flow, driven by an electro-magnetic pump (EMP1), into an upward flow in the inner riser pipe. The schematic of MEGAPIE target general concept and the proton beam entry window, i.e. the hemispherical enclosure of steel LM container are shown in Fig.1.a.). This target window is cooled by an additional by-

* jacek.patorski@psi.ch; phone +41 5631020 60; fax +41 5631031 31; http://asq.web.psi.ch/ASQ/projects/liquid/liquid.html

Thermosense XXIX, edited by Kathryn M. Knettel, Vladimir P. Vavilov, Jonathan J. Miles,

Proc. of SPIE Vol. 6541, 65410C, (2007) · 0277-786X/07/\$18 - doi: 10.1117/12.723283 
pass pipe circuit the so called by-pass pipe nozzle jet flow. The rectangular $20 \times 10 \mathrm{~mm}$ open nozzle-end of the bypass tube forms an LBE jet flow directly streaming onto the inner surface of the window.

Basic geometrical parameters of the assembled lower part of the real target are displayed in Fig.1b.) and represent the principal idea of pumped flow configuration:

- Main (M) flow forced by main electro-magnetic pump (EMP1) transporting LBE to the spallation zone. The Mflow is responsible for recirculation and transporting the strongly heated LBE after spallation to the heat exchanger of the target, which is placed on the top of the whole target system.

- By-pass jet (B) flow forced by by-pass electro-magnetic pump (EMP2) providing a cooling for the hemispherical proton beam entrance window of LM container. The steel wall of the window will be cooled mostly by a LBE bypass nozzle jet B-flow across the window area and additionally by main LBE M-flow.

The MEGAPIE Integral Test (MIT) was an important milestone before the target system has been exposed to the irradiation experiment. Within the MIT we had the unique possibility to investigate the qualitative and quantitative thermohydraulic characteristics of the LM cooling of the window made of, T91 $(9 \mathrm{Cr}-1 \mathrm{MoVNb})$, a martensitic steel, of the real

MEGAPIE target.
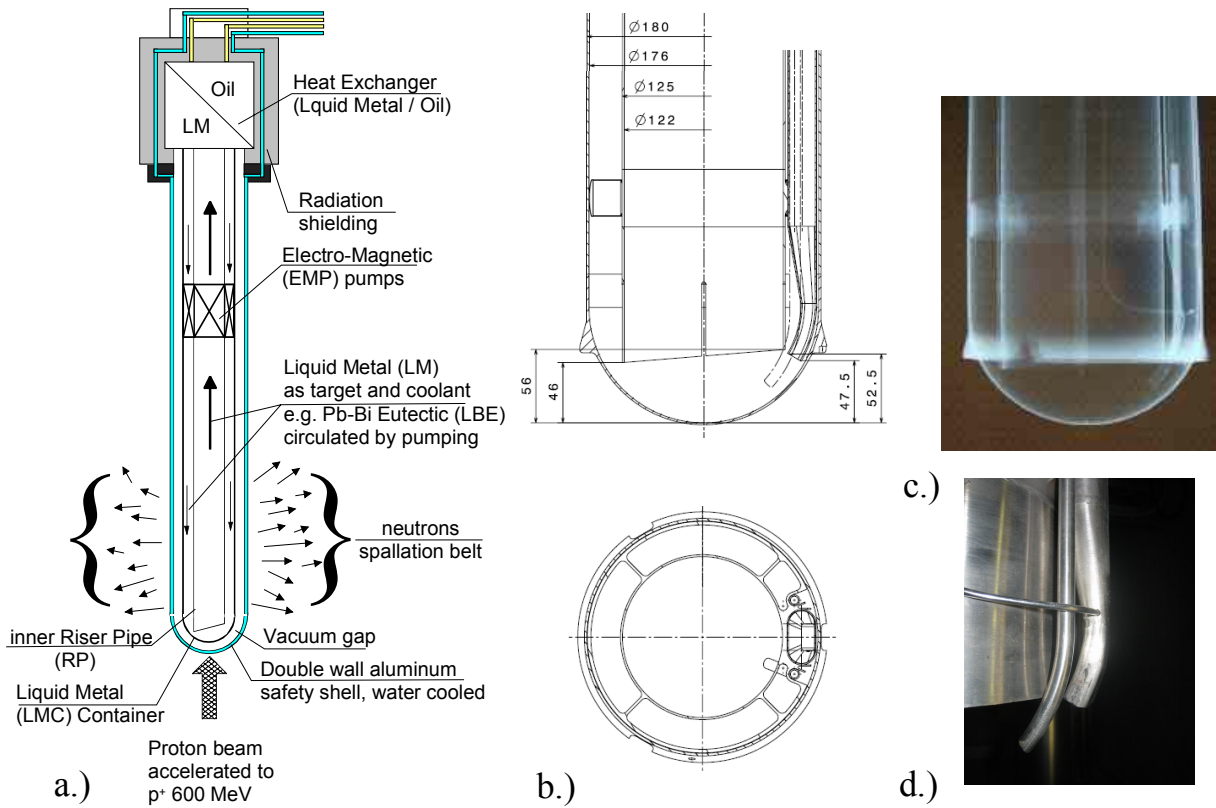

Contrary to the previous thermo-hydraulic window cooling experiments for MEGAPIE, (the so called KILOPIE experiment series, [1], [3], [4] ), all MITMEGAPIE experiment insertion are real finally used structural components. Namely it concerns the following parts in the lower plenum chamber of the LM containers:

i. the riser tube and its slanted ending with slitcuttings,

ii. the by-pass pipe and its ending nozzle,

iii. the fill $\&$ drain pipes and their ending nozzles, iv. the thermocouples (TC) sheath wires,

v. the spacers between LM container and riser pipe, and vi. the geometrical configuration all this parts to each other.

It has big relevance because even small geometrical differ-

Fig.1a.) Schematic of MEGAPIE LM target general concept in vertical configuration at PSI SINQ facility. b.) Geometrical flow configuration in lower part of LM container of the MEGAPIE target. c.) X-ray picture of real LM container of target lower plenum before LBE filling. d.) Photography of insertions details: part of slanted riser, draining pipe, rectangular nozzle of by-pass tube with TC sheath wire.

ences between real manufactured and used parts for the MEGAPIE target in comparison to design dimensions or insertion parts used in pre-experiments (as e.g. KILOPIE) can have influence on the LM flow and finally on the cooling. As well the real target's LM, LBE has been used. At the end of the MIT window cooling experiment series even the real LM container with original window has been used.

Details of the target geometrical configuration in the target's lower plenum are shown in a form of the target's lower part drawings, X-rays and photography of manufactured construction in Fig. 1 b-d.). Just as well we have used the real flow rates (indicated by supplied AC currents in Ampere) forced by real MEGAPIE pumps: EMP1, EMP2 and mix of both pumps according foreseeing regime matrix.

Basically the best cooling effect is expected from the symmetrically spread by-pass jet flow. The symmetry of the flow pattern to the jet axis is desirable but some elements in the lower plenum of the target (as fill and drain pipes and TCs sheath wires) are placed non symmetrically to the axis of the by-pass nozzle jet flow and can disturb the symmetry. The range of the influence of these parts (and eventually other manufacture deficiencies) on the cooling was an important in during the MIT investigation program. 
During MIT we have performed four experiments concerning a sufficiency of the cooling of the target window, in chronological order:

\#1. The MIT-Warm Jet-1 experiment on November 25,. 2005.

\#2. and \#3. The MIT-Warm Jet-2 experiment and the MIT-KILOPIE experiment, both on December 5, 2005.

\#4 The MIT-Warm Jet-3 experiment on December 14, 2005.

The experiments \#1-\#3 are using the original target insertions as mentioned above and special sensor dishes, i.e. small mock-ups of the window(ca. $100 \mathrm{~mm}$ diameter with only $0.5 \mathrm{~mm}$ thick wall), which have been flanged to special LM container. The different sensor specimen dishes have been used to enhance of measurement sensitivity. The original target, with welded LM container, as shown in Fig. 1.b.-d.), and with 1.5-2.0 mm thick original window, has been investigated at the end of the MIT window cooling experiment series, as \#4, and was named MIT-Warm Jet-3 Experiment.

\section{GOALS OF EXPERIMENTAL INVESTIGATIONS}

According to the qualitative and quantitative goals of this experiments series the windows cooling measurement campaigns can be divided into two groups.

The first group of measurements, which mostly has followed the qualitative goals, can be called MIT-Warm Jet Experiments group and consists of three series MIT-Warm Jet-1, -2 and -3 Experiments (chronologically numbered \#1, \#2 and \#4).

The formulation of the goals for this group is:

Visualization of the EMP2 forced LBE B-flow. The visualization has been made using infra red thermography (IRT) measurement techniques.

- Analysis of the geometrical pattern shape of the temperature fields and their dynamic changes during short time of steady common B- and M-flows (IRT data recording time of ca. 1-2 minutes) for different combinations of power supply currents of flow forcing electromagnetic pumps EMP1 and EMP2.

The observation of different infrared radiation intensity of the jet streaming pattern field, for different combinations of power supply currents of EMP1 and of EMP2 as an effect of flows mixing or i.e. a penetration of B-flow into M-flow. The range of the matrix of EMP1- and EMP2-currents is covering proportion of M-flow to B-flow around 12.5. This ratio has been found [5], as critical one for reliably and sufficient cooling of the target window.

In other words the performed MIT-Warm Jet-1, -2 and -3 Experiments have investigated the geometrical temperature distribution on an area of the target steel window as indication for the covering of the proton beam footprint area by the nozzle jet streaming.

The second group, which has mainly followed the quantitative goal, has been named MIT-KILOPIE Experiment. During whole MIT window cooling investigations, chronologically performed as \#3, this group consists of one experimental series, where the DC constant heat flux, established with ca. $1 \mathrm{KW}$ powered sensor in form of hemispherical window's mock-up has been used. For this reason, because this $1 \mathrm{KW}$ power, following the naming style of MEGAPIE (MEGAwattPIlotExperiment), we have named all our previous experiments for the determination of heat transfer coefficient (HTC) and of course including this one; the KILOPIE (KILOwatt PIlot Experiment) experiments.

The formulation of MIT-KILOPIE experiment goal is:

The determinations of HTC fields and their dynamic characteristic i.e. HTC two dimensional distribution and its changes in time using the 2DD-IRT methodology [1].

\section{MIT-WARM JET EXPERIMENTS}

\subsection{Thermal conditions of MIT-Warm Jet Experiments.}

All Warm Jet Experiments derive benefit from the fact, that EMP2 is giving so much heat to the pumped LBE, that the temperature of the B-flow is slightly higher as the temperature of the M-flow.

The visualization of the by-pass tube nozzle jet streaming pattern has been made using IRT measurement techniques. The temperature difference of both flows amounts to $4^{\circ} \mathrm{K}$, and the nozzle jet heats the hemispherical window wall of LBE container. This fact is presented in Fig.2. The localization of thermocouples is shown in Fig.2.a.) : TC085 placed in the nozzle of B-flow and TC074 placed below the edge of the riser pipe.

For example the temperature plot of both TCs in Fig. 2.b.), during the different M-flow and B-flow combinations of MIT-Warm Jet-3 Experiments, shows a quasi constant temperature difference at the temperature level of ca. $240^{\circ} \mathrm{C}$ during the whole measurement time. 

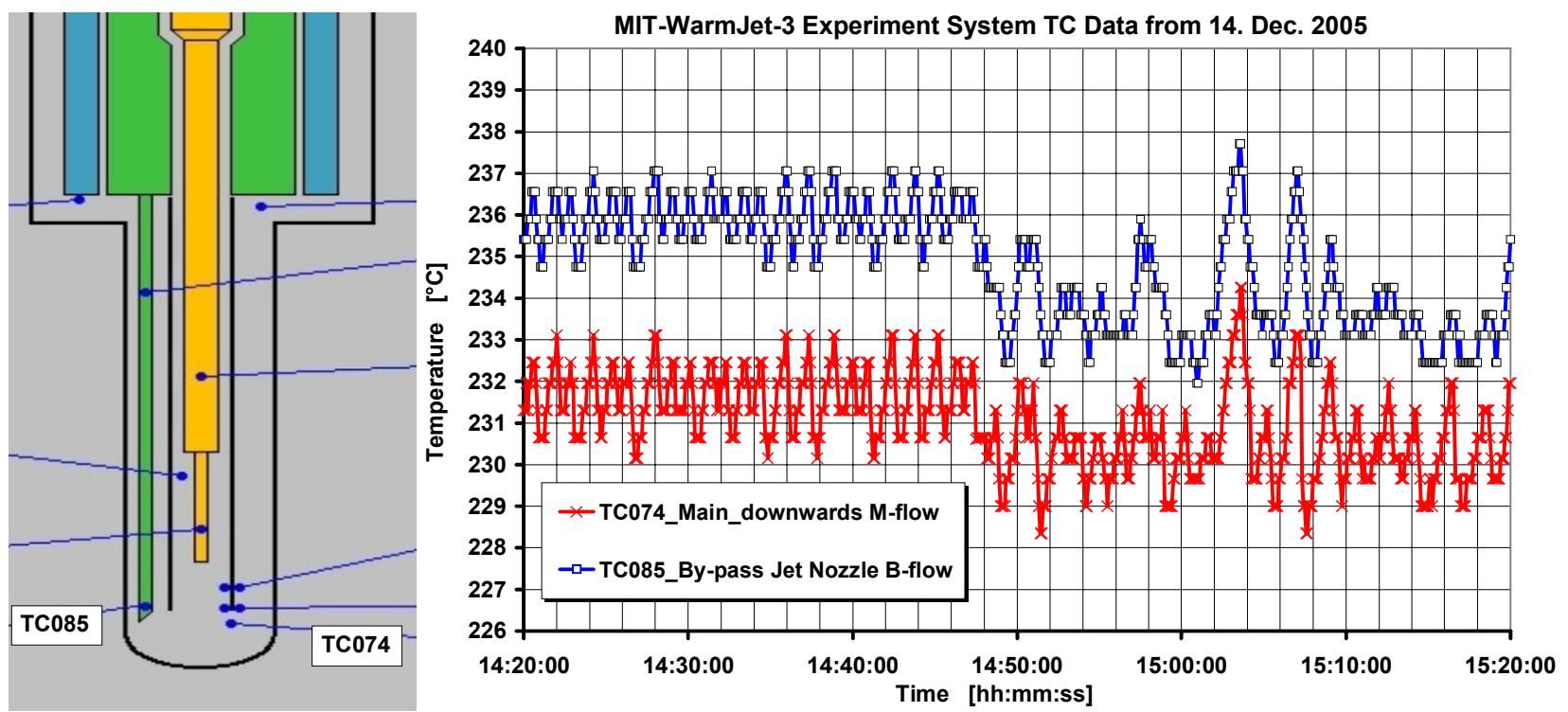

Figure 2a.) Position of MEGAPIE TC085 (by-pass nozzle B-flow) and TC074 (downward M-flow). 2b.) Temperature time plot of TC085 (by-pass nozzle B-flow) and TC074 (downward main M-flow)

\subsection{Set-up and instrumentation of the MIT-Warm Jet-3 Experiment.}

From the whole series of three MIT-Warm Jet Experiments the most interesting is the last one named the MIT-Warm Jet-3 Experiment, because of using, in addition to mentioned original insertion, the real window welded to LM container as it was shown in Fig. 1.b.-d.). The original, used during MEGAPIE irradiation, welded LM container with varying window's wall thickness 1.5-2 mm has been mounted at the end of whole MIT and was only investigated during MIT-Warm Jet-3 experiment. Directly after mounting the measurements of MIT-Warm Jet-3 Experiment the target was transported from MIT stand (MITS) to irradiation place in the SINQ facility.

The set-up schematic is shown in Fig.3.a.) The scanner was placed in the distance of $110 \mathrm{~cm}$ below the window and by using the $20^{\circ}$-lenses the maximum fulfilling of the field of view (FOV) of the THV900 in this distance has been achieved. The uncovering of the window from the rock wool thermal insulation, shown on photographs in Fig.3.b.-c.), had small dimension in comparison to $4 \mathrm{~m}$ long cylinder surface of LM container and has not significant influence on behavior of LBE flow or freezing.

The instrumentation for MIT-Warm Jet-3 Experiment was very simple. We have used for simultaneous measurement two different temperature equipments:

- target's control system thermocouples TC074 and TC085 as shown in Fig.2.) and descripted in section 3.1, and

- IRT scanner of THV900 AGEMA FLIR [6], placed directly below the steel window (Fig.3.a) in such manner that the nozzle of the jet was on the top edge of thermograms.

The TCs measurements have been recorded on system controlling PC and IRT computers for off-line post data evaluation.

The window surface was uncovered by removing of the rock wool thermal insulation, which has been used on the MITS to avoid heat loss and freezing of liquid LBE. During MIT window cooling experiments the average operating temperature of LBE in LM container was about $240^{\circ} \mathrm{C}$ and approximately $100 \mathrm{~mm}$ thick insulation wrapped in aluminum foil was used.

\subsection{The MIT-Warm Jet-3 experimentation.}

To find out, which combination of $\mathrm{M}$ and $\mathrm{B}$ flows is qualitatively optimal from:

- the geometrical covering of the foot print area of the proton beam, defined as concentric circle with diameter 32 $\mathrm{mm}$, and

- the stability of the flow fluctuation behavior,

point of view, a series of 15 combination-cases has been performed. 

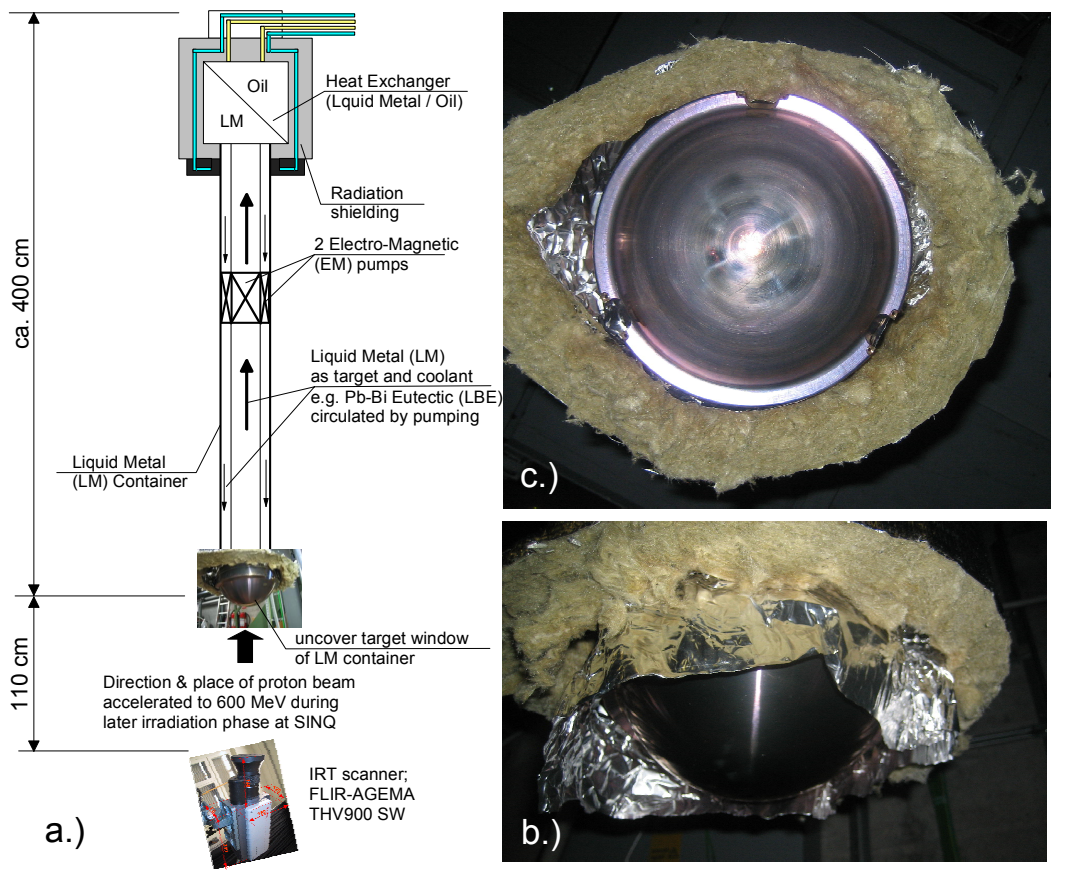

Figure3:

a.) Simplified schematic of set-up of Warm Jet-3 Experiment: axial localization of the FLIR-AGEMA THV900 SW scanner $110 \mathrm{~cm}$ below window.

b.) Uncovering the $240{ }^{\circ} \mathrm{C}$ hot window from rock wool thermal insulation at MITS.

c.) The photography view of the target window as from IRT scanner position.
Table 1 shows the matrix of the MITWarm Jet-3 experiment, where the parametrization of cases is based on the currents supplied to the both EMP1 for Mflow and EMP2 for B-flow. Additional to the measured currents the matrix shows the approximate values of the corresponding flow rates.

The IRT- and TC measurement data recordings for each matrix case consists of two phases performed subsequently in one run

- MB-phase; where the IRT and TCs data acquisition is started at the time of the fully developed, steady state flow for mixed both M-flow and B-flow.

M-phase; where the switching-off of the supply current to the EMP2 the Bflow is stopped. After a while only the stable M-flow is observed, which is few centigrade lower; it could be up to maximally ca. $4^{\circ} \mathrm{K}$ cooler, as expected according section 3.1 and Fig.2.

In Figures 4 and 7 the time temperature plot for the central area of the window shows both phases and characteristic temperature drop after switching-off the Bflow during a typically ca.60 s long IRT recording for case M17B18.

\begin{tabular}{|c|c|c|c|c|c|c|c|c|}
\hline & $\begin{array}{l}\text { EMP2 current } \\
\text { [A] for B-flow }\end{array}$ & 12 & 13 & 15 & 18 & 23 & 28 & 30 \\
\hline $\begin{array}{l}\text { EMP1current } \\
\text { [A] for M-flow }\end{array}$ & $\begin{array}{c}\text { Flow rate } \\
{[\text { liter } / \mathrm{s}]}\end{array}$ & ca. 0.14 & ca. 0.15 & ca. 0.18 & ca. 0.21 & ca. 0.28 & ca. 0.33 & ca. 0.36 \\
\hline 11 & ca. 1.8 & nop & $\begin{array}{c}\text { case } \\
\text { M11B13 }\end{array}$ & notpe & $\begin{array}{c}\text { case } \\
\text { M11B18 }\end{array}$ & $\begin{array}{c}\text { case } \\
\text { M11B23 }\end{array}$ & & $\begin{array}{c}\text { case } \\
\text { M11B30 }\end{array}$ \\
\hline 17 & ca. 2.8 & $\begin{array}{c}\text { case } \\
\text { M17B12 }\end{array}$ & 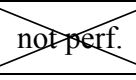 & $\begin{array}{c}\text { case } \\
\text { M17B15 }\end{array}$ & $\begin{array}{c}\text { case } \\
\text { M17B18 }\end{array}$ & $\begin{array}{c}\text { case } \\
\text { M17B23 } \\
\end{array}$ & $\begin{array}{c}\text { case } \\
\text { M17B28 }\end{array}$ & $\begin{array}{c}\text { case } \\
\text { M17B30 } \\
\end{array}$ \\
\hline 23 & ca. 3.7 & & $\begin{array}{c}\text { case } \\
\text { M23B13 }\end{array}$ & & $\begin{array}{c}\text { case } \\
\text { M23B18 }\end{array}$ & $\begin{array}{c}\text { case } \\
\text { M23B23 }\end{array}$ & $\begin{array}{c}\text { case } \\
\text { M23B28 }\end{array}$ & $\begin{array}{c}\text { case } \\
\text { M23B30 }\end{array}$ \\
\hline
\end{tabular}

Table 1: Matrix of MIT-Warm Jet-3 experiment.

\subsection{IRT measurements data evaluation procedure for the MIT-Warm Jet experiments series.}

The raw IRT measurement has been performed with so called "black body" assumption i.e. with the emissivity factor set to 1 for all measurement pixels building the whole thermogram. On the raw thermogram in Fig. 4, from the period of MB-phase we can not recognize small temperature differences caused by the jet of the B-flow because of the nonhomogenously distributed spectral directional emissivity field of the hemispherical polished steel window. For this reason the surface temperatures values of the T91steel window are wrong too. They are at the level of ca. $120{ }^{\circ} \mathrm{C}$ instead of expected ca. $230^{\circ} \mathrm{C}$.

During the M-phase we found by the TCs measurement, that in whole lower plenum of the target constant temperature prevail. We have used this fact for determination of the spectral directional emissivity chart of the window with ERIKA [6] procedure by the assuming equal temperature distribution for the whole window surface during the M-phase flow. The emissivity chart and emissivity profile along the diagonal line is shown in Fig. 5 


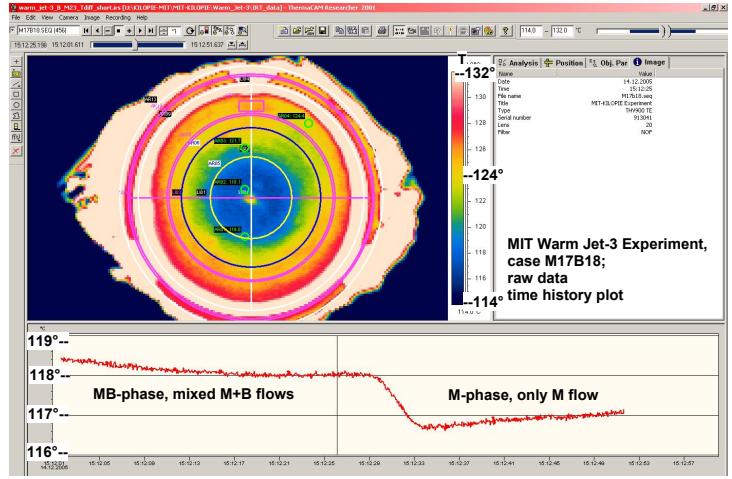

Figure 4: Raw data thermogram from MB-phase flow and time history plot for central area temperature of case M17B18

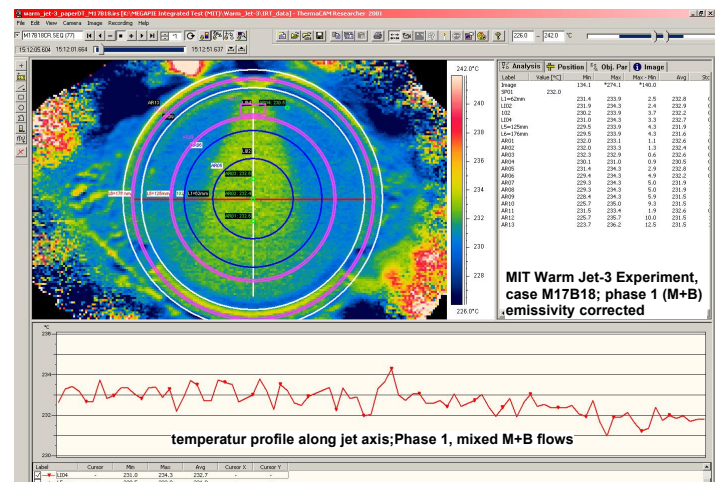

Figure 6: Emissivity corrected thermogram from MB-phase flow and temperature profile along jet axis, case M17B18
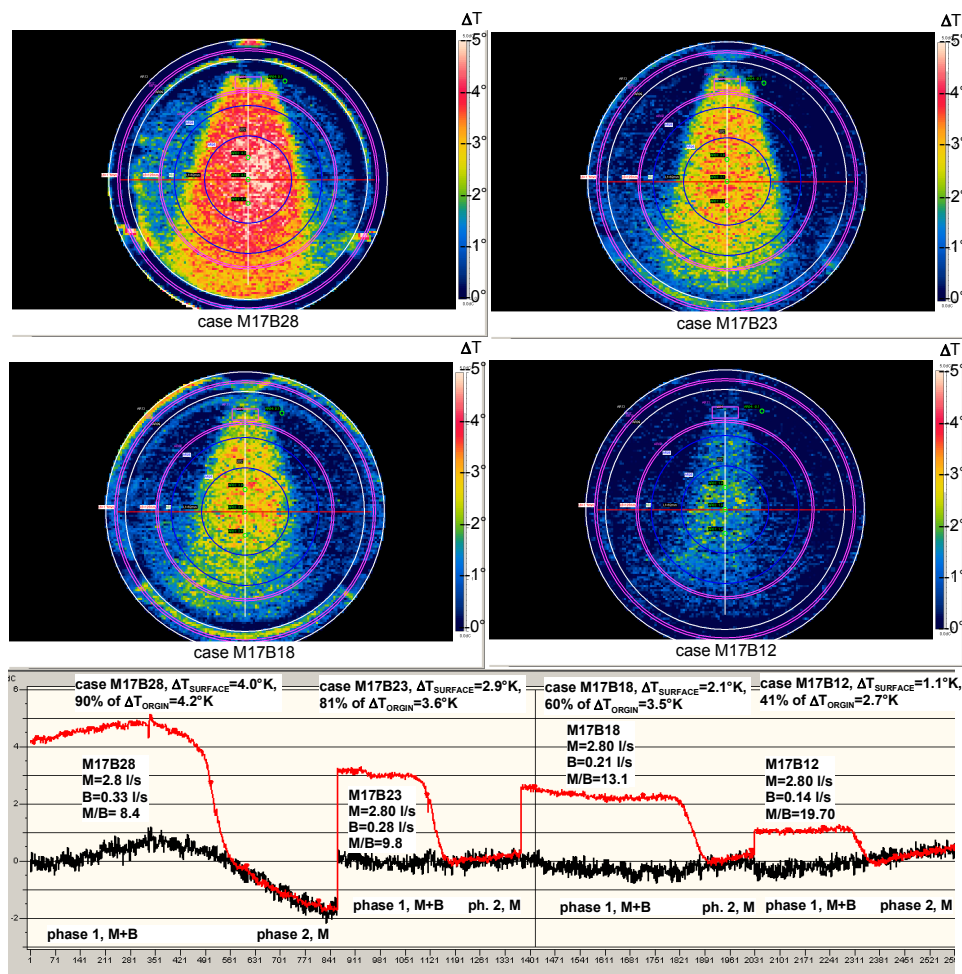

Figure8:Results of some cases from 17 Ampere Series of Warm Jet-3 Exp.

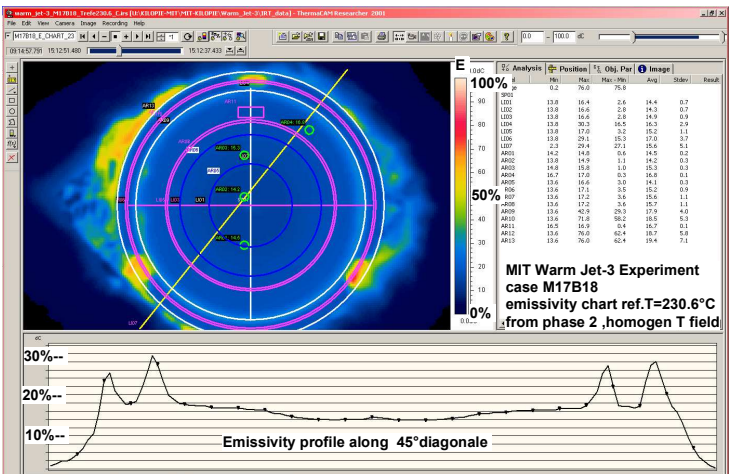

Figure 5: Emissivity chart and emissivity profile of window based on homogenous temperature of M-phase for case M17B18

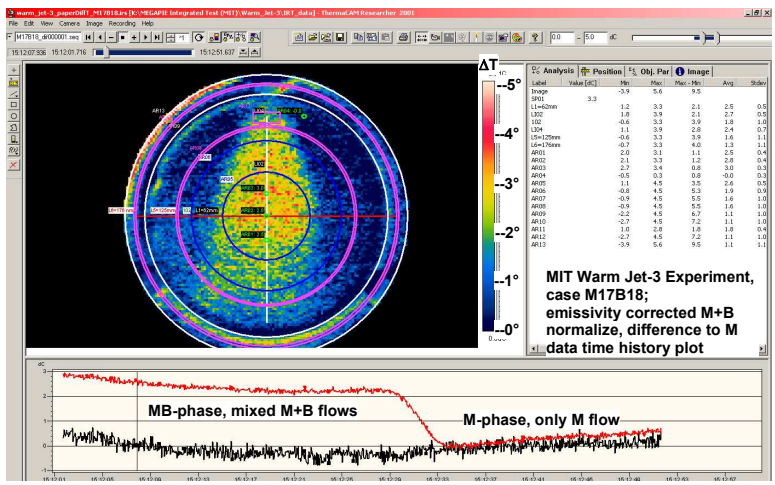

Figure 7: Normalize thermogram of MB-phase for case M17B18

The profile over the emissivity chart is showing a known characteristically directional dependency of the emissivity for metals; in the range from $0^{\circ}$ (i.e. normal direction) to $20^{\circ}$ it is constant (in our case for polished surface of the T91 hemispherical steel shell it amounts to the ca. 14\%), from $20^{\circ}$ to $70^{\circ}$ rises slightly (in our case from $14 \%$ to $18 \%$ ), and over $70^{\circ}$ at grazing angles, the emissivity first increases sharply before dropping back to zero, pp.77-78 in [7].

The obtained emissivity chart has been used for the correction of the raw thermograms. Such a corrected thermogram is shown in Fig. 6. Effectiveness of this correction is amazing, because the true temperature distribution is making the warm jet penetration visible. We can observe pattern of the jet streaming, its gradually widening and the gradually weakening of temperature through cumulative mixing of the warm Bflow in the slightly colder M-flow.

From thermo hydraulics point of view it is the very important information, that the influence of the jet is present on the inner sur- 
face of the window. The kinetic energy of the low B-flow rate of the jet was enough strong to penetrate the M-flow with essentially higher flow rate and reach the window surface. We can admit, that when the jet has reached the surface, his higher linear velocity, even partially, but is present in boundary layer on the window and consequently the cooling in this area is better then in a rest-area, where the significantly slower M-flow is touching the window inner surface. Although the qualitative character of this information, we can derivate some interesting relative numbers. For example the profile along the jet axis line in Fig. 6 is showing at the beginning from rectangular jet outlet to the center of the window almost constant temperature of ca. $233^{\circ} \mathrm{C}$, but behind the center the temperature decreases slightly to. $231.5^{\circ} \mathrm{C}$ and when we know for the M17B18 case the output jet temperature $\mathrm{T}_{\mathrm{B}}=234.2^{\circ} \mathrm{C}$ and main flow bulk temperature $\mathrm{T}_{\mathrm{M}}=230.6^{\circ} \mathrm{C}$ we can conclude, that from system origin $\Delta \mathrm{T}_{\text {ORIGIN }}=\mathrm{T}_{\mathrm{B}}-\mathrm{T}_{\mathrm{M}}=3.6^{\circ} \mathrm{K}$ to the window surface $\Delta \mathrm{T}_{\text {SURFACE }}=\mathrm{T}_{\text {SURFACE}}-\mathrm{T}_{\mathrm{M}}$, varying from $2.4^{\circ} \mathrm{K}$ to $0.9^{\circ} \mathrm{K}$ is coming through i.e. only from ca $60 \%-20 \%$ of initial value. Such relative comparison between different cases from matrix in Table 1 can be done easier when the temperature absolute values are normalize to the M-flow bulk temperature $T_{M}$ (individually for each case), this temperature changes periodically within each case and between cases, because the characteristics of the three-way-valve of the target heat exchanger.

In Fig. 7 such normalized thermogram for the case M17B18 is shown, where additionally the non interesting parts of insulation are cut off. In Fig.7 below are shown time history plots: for the M-flow bulk temperature (black line) from small surface, which is not affected by B-flow during the MB-phase, and for the central $60 \mathrm{~mm}$ diameter area of the window (red line). The previously mentioned characteristic of heat exchanger can be recognized through non constant value of M-flow bulk temperature, black plot line in Fig.7. The concentric double line circles (violet color) seen in the thermograms represent the diameters and thickness of riser pipe (outer/inner diameter of 122/125 mm) and LM container cylinder $(176 / 180 \mathrm{~mm})$, the rectangular area on the vertical symmetry axis above on the outer side of riser pipe, represents the projection of 10x20 mm nozzle outlet of the by-pass. In the next section 3.4 the presentation of results will based on normalized thermograms like this from Fig.7.

\subsection{Results of MIT-Warm Jet Experiments series}

The results of evaluated measurements are basically presented in form of normalized IR thermograms (like shown in Fig.7) or normalized IRT thermograms sequences, worked out from the raw temperature field thermograms as described in section 3.3. The normalized thermograms allow direct comparison between different cases in geometry and intensity of penetration because the origin difference of B-flow an M-flow vary slightly around of $4^{\circ} \mathrm{K}$, see Fig.2. From numerical data of recorded thermograms each kind of additional evaluation, only limited by geometrical- and time resolution features of THV 900 device, can be done. For example following evaluation types are possible: the statistics evaluations, the profiles along arbitrary defined lines or time plots for arbitrary defined point or areas of interest and etc. Because of limited place for this paper we are presenting, as typical example, only part of results in form of thermograms from performed cases from matrix in Tab.1. In Fig.8 the normalized thermograms for four cases of 17 Ampere series for current of EMP1 (M-flow) have been chosen sequentially with ca. 5 Ampere steps of current for EMP2 (B-flow) namely 28, 23, 18, and 12 Ampere.

The visualization of the jet flow pattern shows, as expected, weaker penetration of the warm jet to the window surface, with lower currents of EMP2, i.e. lower B-flow rates. The visualization of the flow pattern allows the conclusion that the proton beam footprint is well covered by the jet flow in nearly all cases of the Tabl.1 matrix. The footprint is characterized by the $2 \sigma$ radius (ca. $32 \mathrm{~mm}$ ) of the rtation-symmetric Gaussian distribution.

For few combination cases of 11 Ampere series for power supply current of EMP1 (M-flow with weak flow rate of ca.1.8 1/s) and the stronger power supply currents of 30 and 23 Ampere for EMP2 (B-flows with flow rates of ca. 0.36 and $0.28 \mathrm{l} / \mathrm{s}$ ) a low frequency, ca. $0.15 \mathrm{~Hz}$, fluctuation of the jet pattern with amplitude of ca. $10 \mathrm{~mm}$ has been found. Even for these situations of non-stable flow combinations the $2 \sigma$ area has been covered by the jet flow. This phenomenon will be shown in an animation during presentation of the paper. The relevance of this phenomenon is, that the central footprint area of the proton beam at the window should be always covered by the jet pattern.

The analysis of thermograms also revealed a slight asymmetry of the jet temperature field pattern but not relevant from foot print covering point of view.

\section{MIT-KILOPIE EXPERIMENTS}

\subsection{Set-up and instrumentation of MIT-KILOPIE experiment.}

Because of the quantitative goal of this part of MIT window cooling experiments, namely the determination of HTC, we have used another set-up. The active small sensor dish as the mock-up of the central part of the window has to be used. This sensor dish is PSI's own development and has been described in [1]. The sensor was mounted to the lower part of the flanged LM container, which has been used at MITS for all MIT experiments (with exception of in section 3 reported MIT-Warm Jet-3 experiment) with special triple flange interface vessel. In Fig. 9 the longitudinal- and cross- 
section of target's lower part drawings and schematic of the whole set-up for MIT-KILOPIE experiment are shown. The details of the used 2DD-IRT instrumentation and methodology are described in [1]. We want to put attention on other aspects of this application.

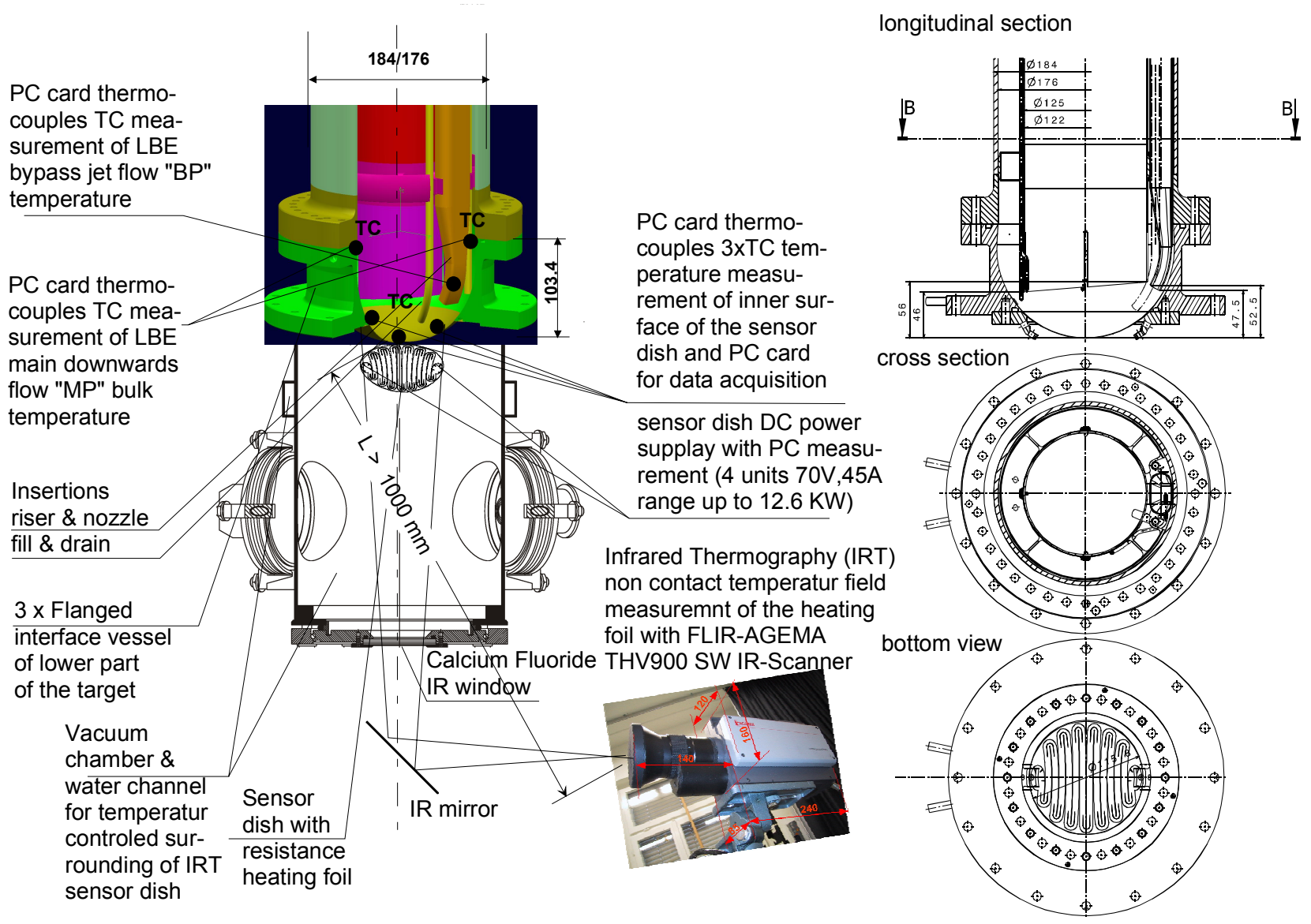

Figure 9:

a.) MIT-KILOPIE experiment set-up and instrumentation. b.) Sections and bottom view of target's lower plenum for MIT-

KILOPIE experiment.

\subsection{The MIT-KILOPIE experimentation.}

For the determination of HTC values and their distribution we have perform a bigger matrix of 30 combination cases shown in Tab. 2.

\begin{tabular}{|c|c|c|c|c|c|c|c|c|c|}
\hline & $\begin{array}{l}\text { EMP2 current } \\
\text { [A] for B-flow }\end{array}$ & 12 & 15 & 18 & 21 & 24 & 26 & 28 & 30 \\
\hline $\begin{array}{l}\text { EMP1current } \\
\text { [A] for M-flow }\end{array}$ & $\begin{array}{c}\text { Flow rate } \\
{[\text { liter } / \mathrm{s}]}\end{array}$ & ca. 0.14 & ca. 0.18 & ca. 0.21 & ca. 0.25 & ca. 0.29 & ca. 0.31 & ca. 0.33 & ca. 0.36 \\
\hline 11 & ca. 1.8 & $\begin{array}{c}\text { case } \\
\text { M11B12 }\end{array}$ & $\begin{array}{c}\text { case } \\
\text { M11B15 }\end{array}$ & $\begin{array}{c}\text { case } \\
\text { M11B18 }\end{array}$ & $\begin{array}{c}\text { case } \\
\text { M11B18 }\end{array}$ & $\begin{array}{c}\text { case } \\
\text { M11B23 }\end{array}$ & $\begin{array}{c}\text { case } \\
\text { M11B26 }\end{array}$ & $\begin{array}{c}\text { case } \\
\text { M11B28 }\end{array}$ & $\begin{array}{c}\text { case } \\
\text { M11B30 }\end{array}$ \\
\hline 17 & ca. 2.8 & $\begin{array}{c}\text { case } \\
\text { M17B12 }\end{array}$ & $\begin{array}{c}\text { case } \\
\text { M17B15 }\end{array}$ & $\begin{array}{c}\text { case } \\
\text { M17B18 }\end{array}$ & $\begin{array}{c}\text { case } \\
\text { M17B18 }\end{array}$ & $\begin{array}{c}\text { case } \\
\text { M17B23 }\end{array}$ & $\begin{array}{c}\text { case } \\
\text { M17B26 }\end{array}$ & $\begin{array}{c}\text { case } \\
\text { M17B28 }\end{array}$ & $\begin{array}{c}\text { case } \\
\text { M17B30 }\end{array}$ \\
\hline 22 & ca. 3.6 & $\begin{array}{c}\text { case } \\
\text { M22B12 }\end{array}$ & $\begin{array}{c}\text { case } \\
\text { M22B15 }\end{array}$ & $\begin{array}{c}\text { case } \\
\text { M22B18 }\end{array}$ & $\begin{array}{c}\text { case } \\
\text { M22B18 }\end{array}$ & $\begin{array}{c}\text { case } \\
\text { M22B23 }\end{array}$ & $\begin{array}{c}\text { case } \\
\text { M22B26 }\end{array}$ & $\begin{array}{c}\text { case } \\
\text { M22B28 }\end{array}$ & $\begin{array}{c}\text { case } \\
\text { M22B30 }\end{array}$ \\
\hline 30 & ca. 4.9 & $\begin{array}{c}\text { case } \\
\text { M30B12 }\end{array}$ & $\begin{array}{c}\text { case } \\
\text { M30B15 }\end{array}$ & $\begin{array}{c}\text { case } \\
\text { M30B18 }\end{array}$ & & $\begin{array}{c}\text { case } \\
\text { M22B30 }\end{array}$ & & & $\begin{array}{c}\text { case } \\
\text { M30B30 }\end{array}$ \\
\hline
\end{tabular}

Table 2: Matrix of MIT-KILOPIE experiment. 
Each case has the same characteristic and consists of 4 phases as can be seen on an example of the temperature plots of three TCs on the inner surface of the dish. The simultaneous recording of the TC and dish voltage on PC and IRT data is starting at the moment of fully developed common main- and by-pass-flow in the MB-phase.

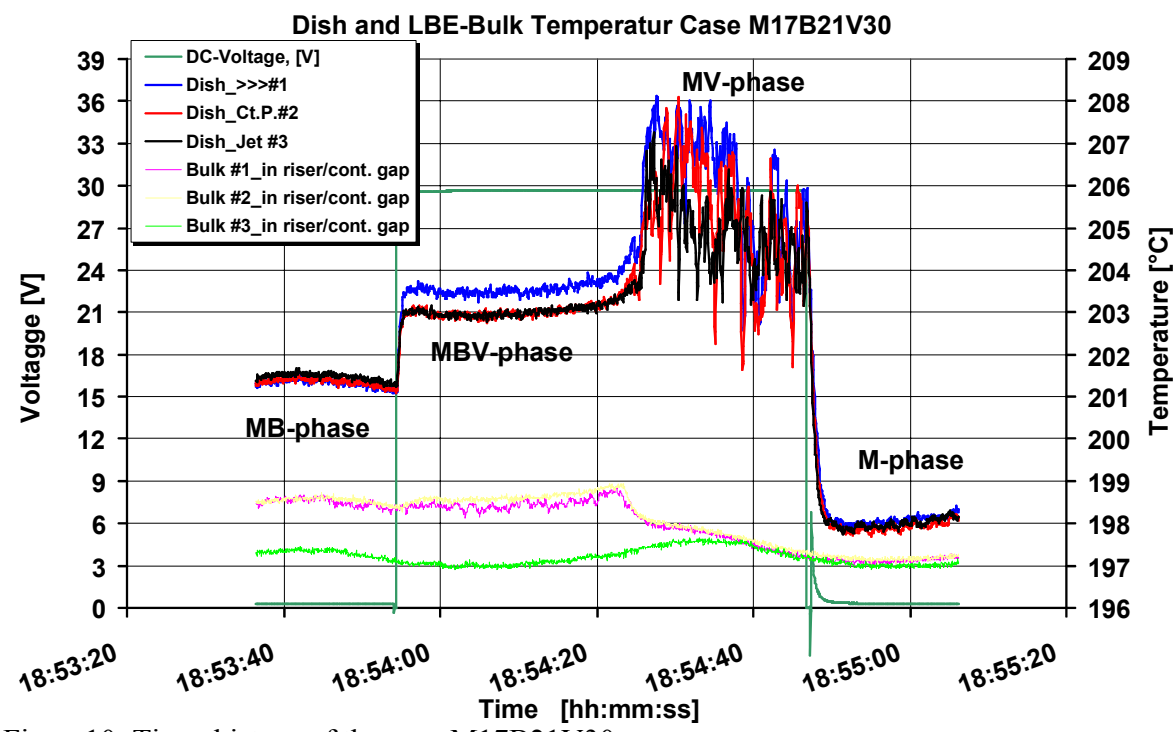

For the period of the same flow conditions for both $\mathrm{M}$ flow and B-flow in the next MBV-phase additionally the heat flux power input from the sensor, after switching on the $30 \mathrm{~V}$ DC current on the sensor dish heater, is causing a rise of the temperature. In the next characteristic MV-phase of temperature plot the by-pass Bflow is switched off. In this MV-phase we can observe once more a rise of temperature caused by worse cooling in absence of the jet streaming. Finally in the M-phase, after switching off the $30 \mathrm{~V}$ DC current supplying of the sensor, the temperature of the sensor dish

Figure10: Time-history of the case M17B21V30

dropped rapidly to the bulk temperature of the M-flow. From each of these phases we have to extract the necessary information for the determination of the HTC field values from IRT measurements.

\subsection{IRT measurement data evaluating procedure for the MIT-KILOPIE experiment.}

As know from our previous works [1] for the determination of HTC from IRT data the evaluation is based on the Eq. 4.1, which allow calculate HTC for each pixel of IRT thermogram measured.

$$
H T C=\frac{q^{*}}{T_{\text {out }, \text { foil }}-q^{*} \cdot R_{\text {th }}-T_{\text {bulk }}}
$$

The HTC is expressed with values measured during the KILOPIE IRT Experiment, where: $q^{*}$ is applied heat flux on the sensor dish, $T_{\text {out foil }}$ is the emissivity corrected outer surface temperature of the sensor during the applying of heat flux, $T_{\text {bulk }}$ is bulk field temperature of the cooling flow in absence of heat flux and $R_{t h}$ is thermal resistance chart of the sensor dish.

We will accentuated, that all members of denominator of Eq.4.1 can be obtained as IRT temperature fields during described IRT measurement. Therefore we can write Eq.4.2

$$
H T C=\frac{q^{*}}{\left(T_{\text {out }, \text { foil }}-T_{\text {bulk }}\right)-\left(T_{\text {out }, \text { foil }}^{\text {R test }}-T_{\text {inn }, \text { steel }}^{R \text { test }}\right)}
$$

where additionally the temperature values from thermal resistance test for determination of thermal resistance chart of the sensor dish are present. During such test the constant homogeneous cooling conditions of the sensor have to be realized and after applying of known $q^{*}$ the outer und inner surface temperature field have to be measured.

The Eq.4.2 shows that during IRT data evaluation the local heat transfer coefficient can be constructed from one final difference temperature field, which can be obtain from two temperature difference fields grouped in the denominator of Eq.4.2. The final arithmetic to achieve the local $H T C$ values building the $H T C$ chart is the multiplication of the reciprocals of this final temperature difference field by the constant value of applied heat flux $q^{*}$.

Below we describe how the data measured during above defined phases can be used for the construction of the thermograms from Eq.4.2.

- Preparation of emissivity corrected IRT sequence.

1. Selection of thermocouple TC measured bulk temperature TBM from the beginning of the M phase (main flow only) as $1 \mathrm{~s}$ average value.

2. Creation of emissivity non-corrected (raw measurement data) reference thermogram of main flow bulk temperature field TBM for the same time point and period of $1 \mathrm{~s}$ as for TC measurement in Pt.1. 
3. Creation of IRT emissivity corrected sequence from IRT raw sequence based on reference thermogram from Pt.2

4. Creation of corresponding emissivity chart image for documentation.

- Determination of the thermal resistance correction term = product of thermal resistance chart of the sensor dish and applied heat flux (Determination of the second term of differential thermogram of the HTC equation)

Selection of the thermocouple TC measured thermal resistance experiment temperature TR from second half of the MV phase (phase of main flow and applied heat flux q*, symbol V because presence of DC Voltage on the sensor) and building the final TR correction thermogram.

5. Fixing of the time period for the thermal resistance determination from time point after sure demixing of B-flow from M-flow during beginning of MV-phase to time point before the end of MV phase from TCs time history plot.

6. Calculating of average $\mathrm{TC}$ value for TR, from this period

7. Calculating the corrected dish inner surface temperature from the tip of $\mathrm{TC}$ to the wall in distance of $\mathrm{TC}$ wire half diameter.

8. Creation of uniform thermogram image of temperature Pt.7 from non emissivity corrected raw thermogram.

9. Creation of average thermogram of outer surface over the same period from Pt.5 as for TC averaging in Pt.6 from MV phase corresponding to temperature from Pt,7 from emissivity corrected sequence from Pt.3.

10. Creation of the second term difference thermogram, which correspond to product of $\mathrm{q}^{*} \mathrm{x}$ TR chart= Pt.9 thermogram minus Pt. 8 thermogram. (This thermogram is necessary for the creation of final temperature difference of inner surface temperature and 2 bulk temperatures fields: one for the $\mathrm{M}$ phase: TBM and second for the MB phase: TBJ).

- Determination of the first term differential thermograms of the HTC differential thermogram equation (outer surface temperature field - bulk temperature field).

- Procedure for the MBV-phase thermogram minus TBJ bulk temperature field from the MB phase

11. Fixing of half second long period short before end time point of MB-phase for TBJ bulk field for MBV-phase flow from TCs time history plots.

12. Creation of the avg. emissivity corrected thermogram of TBJ by-pass jet flow bulk temperature field for Pt.11 period from MB-phase (used for the determination of HTC during the MBV-phase) from emissivity corrected sequence achieved in Pt.3.

13. Fixing of half second long period after and close to the start time point of the MBV-phase from TC time history plot.

14. Creation of the avg. emissivity corrected thermogram proper for MBV-phase for the Pt.13 period, i.e. for the beginning of MBV phase, (used for the determination of HTC during the MBV-phase) from emissivity corrected sequence achieved in Pt.3.

15. Creation of the first term differential thermogram proper for MBV phase=Pt.14 thermogram minus Pt. 12 thermogram.

- Procedure for the MV-phase thermogram minus TBM bulk temperature field from M-phase.

16. Fixing of half second long period after begin and close to start of M-phase for TBM bulk field for MV-phase flow from TCs time history plot.

17. Creation of the avg. emissivity corrected thermogram of TBM main flow bulk temperature field for Pt.16. period, from phase M, (used by determination of HTC during the MV-phase), from emissivity corrected sequence achieved in Pt.3.

18. Fixing of half second long period before and close to the end of the MV-phase from TC time history plot

19. Creation of the avg. emissivity corrected thermogram proper for MV-phase, for the Pt.18 period i.e. before the end of MV-phase, (used by determination of HTC during the MV-phase) from emissivity corrected sequence achieved in Pt.3

20. Creation of the first term differential thermogram proper for MV-phase=Pt.19 thermogram minus Pt.17 thermogram.

- Determination of the final differential thermograms of the HTC differential thermogram equation (inner surface temperature - bulk

- Procedure for the MBV-phase

21. Creation of the final differential thermogram of differential thermograms (first term - second term) proper for MBV phase=Pt.15 thermogram minus Pt.10 thermogram. 
- Procedure for the MV-phase

22. Creation of the final differential thermogram of differential thermograms

(first term - second term) proper for MV-phase=Pt.20 thermogram minus Pt.10 thermogram.

\subsection{Results of MIT-KILOPIE Experiment}

As an example from 30 performed cases the results of case M17B21 are presented in Fig. 11. The thermograms represent the difference temperature between inner surface and bulk temperature as mentioned in section 3.3 . The $80 \mathrm{~mm}$ diameter circle thermograms are showing only the small part of the window, the whole window has ca. $180 \mathrm{~mm}$ diameter, because of the small ca $100 \mathrm{~mm}$ diameter dimension of the sensor dish. The inside circles indicated the proton beam footprint area and have $32 \mathrm{~mm}$ and $60 \mathrm{~mm}$ diameters respectively.

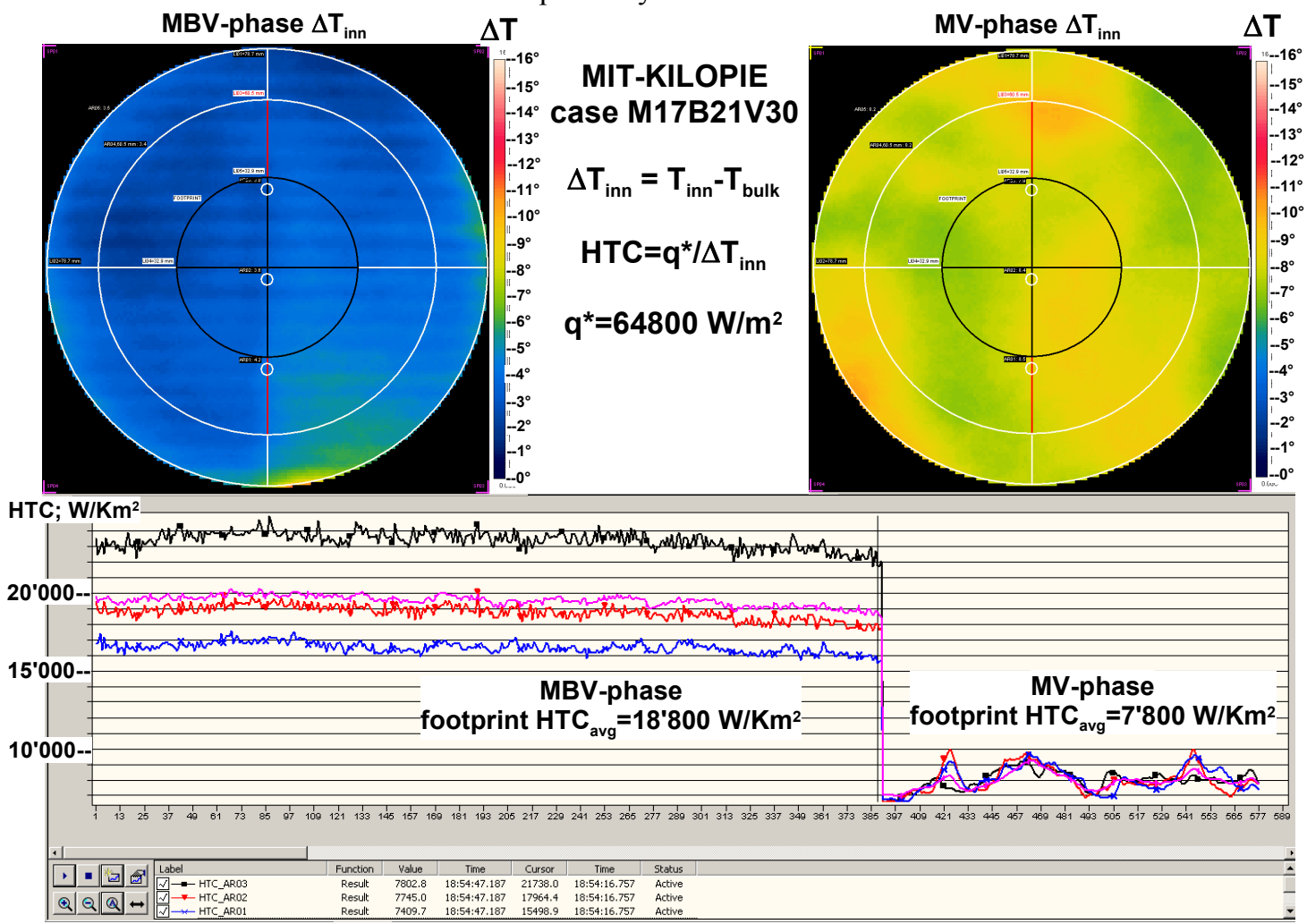

Figure 11: $\Delta \mathrm{T}_{\mathrm{inn}}$ thermograms and HTC plots for case M17B21V30

On the left side the thermogram of the good and very stable cooled MBV-phase is shown, where both the by-pass jet B-flow and main M-flow are present, on the right side the thermogram of poorly cooled MV-phase, where only main flow is present. Below the corresponding time-history plot of short sequences are put together in one diagram. The determined values of HTC for three points on the vertical diameter of $32 \mathrm{~mm}$ (inner circle for the foot print of the proton beam) along the jet axis are plotted. The higher HTC values (black line) correspond to the point, which is closer to the jet outlet on the top of thermograms. The red line plot belongs to the central point and the blue one belongs to the lower point. The average value for the $32 \mathrm{~mm}$ diameter circle of foot print is plotted with violet line. During the MBV-phase the HTC average value for the foot print area amounts to $19000 \mathrm{~W} / \mathrm{Km}^{2}$, the HTC difference along this short distance profile of $32 \mathrm{~mm}$ amounts to ca. $4000 \mathrm{~W} / \mathrm{Km}^{2}$. On the right side the difference thermogram of MV-phase shows essentially smaller HTC values of ca. $8700 \mathrm{~W} / \mathrm{Km}^{2}$ and the behavior of the cooling is not stable. Within one thermogram the relative differences amount up to $4000 \mathrm{~W} / \mathrm{Km}^{2}$ too but the plotted values show bigger fluctuations in time and the cooling is not stable. The animation of this behavior will be presented at the conference. For the reader interested in thermo hydraulics result of the whole series the summarized results of MIT-KILOPIE experiment are shown in Tab.3. They confirm results obtained from previous experiments performed on models or mock-ups of the target. As well the numerical values obtained for some theoretical models are partially confirmed [8]. The temperature profiles along the jet line show only slightly changes. The temperatures differences profiles or HTC profiles show stronger degradation after passing by of the central point of the window. The different degradation of the cooling is evident but the detachment of the jet for the cases with $\mathrm{M} / \mathrm{B}$ flow ratio above 12.5 observed in [5] has not been found. 


\begin{tabular}{|c|c|c|c|c|c|c|c|c|c|c|}
\hline & & BP Current [A] & 12 & 15 & 18 & 21 & 24 & 26 & 28 & 30 \\
\hline & & BP Flow-Rate [l/s] & 0.14 & 0.18 & 0.21 & 0.25 & 0.29 & 0.31 & 0.33 & 0.36 \\
\hline $\begin{array}{l}\text { MP Curr. } \\
\text { [A] }\end{array}$ & $\begin{array}{l}\text { MP Flow- } \\
\text { Rate [l/s] }\end{array}$ & & \multicolumn{8}{|c|}{ MF/BF ratio and Heat Transfer Coefficients (HTC) } \\
\hline \multirow{6}{*}{11} & \multirow{6}{*}{1.8} & ratio M/B (main to by-pass jet flows) & 12.7 & 9.9 & 8.5 & 7.1 & 6.1 & 5.7 & 5.4 & 4.9 \\
\hline & & Pt.\#3 (jet side, $16 \mathrm{~mm}$ to C.Pt.) & 15766 & 16788 & 17514 & 18409 & 18783 & 19459 & 20187 & 20971 \\
\hline & & Pt.\#2 (center, C.Pt.) & 15429 & 16119 & 17280 & 17753 & 17900 & 18947 & 19343 & 20313 \\
\hline & & Pt.\#1 (16 mm behind of C.Pt.) & 13144 & 13642 & 14661 & 15283 & 15429 & 16160 & 16573 & 17802 \\
\hline & & Avg.Pts \#1,\#2,\#3; diam. 32mm & 14780 & 15516 & 16485 & 17148 & 17371 & 18189 & 18701 & 19695 \\
\hline & & main flow only, no jet cooling & 5719 & 5822 & 6095 & 5669 & 5524 & 5796 & 5745 & 5478 \\
\hline \multirow{6}{*}{17} & \multirow{6}{*}{2.8} & ratio M/B (main to by-pass jet flows) & 19.7 & 15.3 & 13.1 & 11.0 & 9.5 & 8.9 & 8.4 & 7.7 \\
\hline & & Pt.\#3 (jet side, $16 \mathrm{~mm}$ to C.Pt.) & 17188 & 17514 & 19636 & 19343 & 19756 & 20124 & 20250 & 20442 \\
\hline & & Pt.\#2 (center, C.Pt.) & 16405 & 17143 & 18462 & 18567 & 18621 & 19115 & 19577 & 19636 \\
\hline & & Pt.\#1 (16 mm behind of C.Pt.) & 13279 & 13966 & 15652 & 15961 & 15921 & 17053 & 17514 & 17326 \\
\hline & & Avg.Pts \#1,\#2,\#3; diam. 32 mm & 15624 & 16208 & 17917 & 17957 & 18099 & 18764 & 19114 & 19135 \\
\hline & & main flow only, no jet cooling & 7902 & 7509 & 7970 & 7970 & 7874 & 7097 & 7687 & 7922 \\
\hline \multirow{6}{*}{22} & \multirow{6}{*}{3.6} & ratio M/B (main to by-pass jet flows) & 25.4 & 19.8 & 17.0 & 14.2 & 12.3 & 11.5 & 10.8 & 9.9 \\
\hline & & Pt.\#3 (jet side, $16 \mathrm{~mm}$ to C.Pt.) & 17851 & 18253 & 19696 & 19343 & 20703 & 20769 & 20903 & 21176 \\
\hline & & Pt.\#2 (center, C.Pt.) & 16919 & 17326 & 18947 & 19115 & 19401 & 19577 & 19938 & 20000 \\
\hline & & Pt.\#1 (16 mm behind of C.Pt.) & 13729 & 14432 & 15961 & 15728 & 16241 & 16744 & 17373 & 17901 \\
\hline & & Avg.Pts \#1,\#2,\#3; diam. 32 mm & 16166 & 16670 & 18201 & 18062 & 18782 & 19030 & 19405 & 19692 \\
\hline & & main flow only, no jet cooling & 9088 & 8276 & 8223 & 8329 & 8438 & 8493 & 8383 & 8276 \\
\hline \multirow{6}{*}{30} & \multirow{6}{*}{4.9} & ratio $M / B$ (main to by-pass jet flows) & 34.6 & 26.9 & 23.1 & & 16.7 & & & 13.5 \\
\hline & & Pt.\#3 (jet side, $16 \mathrm{~mm}$ to C.Pt.) & 17373 & 18621 & 19459 & & 21386 & & & 22898 \\
\hline & & Pt.\#2 (center, C.Pt.) & 16919 & 18153 & 18352 & & 20703 & & & 22116 \\
\hline & & Pt.\#1 (16 mm behind of C.Pt.) & 14965 & 14965 & 15140 & & 16919 & & & 18620 \\
\hline & & Avg.Pts \#1,\#2,\#3; diam. 32 mm & 16419 & 17246 & 17650 & & 19669 & & & 21211 \\
\hline & & main flow only, no jet cooling & 9488 & 10401 & 10077 & & 9488 & & & 10237 \\
\hline
\end{tabular}

Table 3: Heat Transfer Coefficient $\left[\mathrm{W} /{ }^{\circ} \mathrm{Km}^{2}\right]$ values measured during MIT-KILOPIE experiment

\section{CONCLUSIONS}

The MIT-Warm Jet experiments have shown the presence of jet cooling on the window in all combinations of main Mflow and by-pass B-flow. The combination of M23B23, which has been used in the successfully irradiated target has according to interpolation from values of experiments, fulfilled all loads conditions. The covering of the footprint area of the proton beam has been fulfilled for the majority of cases. The heat flux of $64800 \mathrm{~W} / \mathrm{m}^{2}$ used during MIT-KILOPIE experiment supplied to the sensor dish should be higher because of the low accuracy of the measurements due to the small temperature differences in the IRT data evaluation. The dynamic visualization of the cooling has shown low frequency fluctuations $(0.15 \mathrm{~Hz})$ with $10 \mathrm{~mm}$ amplitude for some cases (M11B26, M11B28 and M11B30). The Development Project group of Spallation Neutron Source Division at PSI will apply and improve the 2DD-IRT measurement techniques for development a new high power target in the next future.

\section{REFERENCES}

1. J.A. Patorski, F. Groeschel, "Experimental determination of local convection heat transfer coefficient field using two-dimensional and dynamic infrared thermography (2DD-IRT) method", Proceedings of SPIE, Thermosense XXVIII, Vol. 6205, (ISSN 0277-786X) , Jonathan J. Miles, G. Raymond Peacock, Kathryn M. Knettel, Editors, SPIE-, Bellingham, Washington 98227-0010 USA, April 2006

2. G.S.Bauer, M. Salvatores and G.Heusener, "MEGAPIE, a 1 MW Pilot Experiment for a Liquid Metal Spallation Target", J. Nucl. Mat. 296, pp.17-35, 2001

3. J.A. Patorski, B.Sigg, I. Platnieks, F. Groeschel and R. Stieglitz "Results of IRT- and TC-KILOPIE-1 Experiments“, PSI Annual 2004, ISSN 1423-7326, Vol.III, p.204-205, Switzerland, March 2005

4. J.A. Patorski, F. Groeschel, F. Barbagallo and I. Platnieks, "First results of the KILOPIE-2 Pre-experiment", Proc. 6th Technical Review Meeting, MEGAPIE, SCK-CEN, Mol, Begium, 27-29 ${ }^{\text {th }}$ June 2005

5. M.Daubner, A.Batta, F.Fellmoser, C.-H. Lefhalm, R.Stieglitz, "Turbulent Thermal Mixing of a Heavy Liquid Metal Flow in aTarget Window Geometry - Heatd Jet Experiment" Forschungszentrum Karlsruhe, Wissenschaftliche Berichte FZKA 7098, Germany, September 2005

6. AGEMA Infrared Sytems, "Thermovision ${ }^{\circledR 900}$ Series User's Manual" AGEMA Infrared Sytems Publication No.556960, Sweden, 1993

7. M.F. Modest, "Radiative heat transfer", (First Edition), McGraw-Hill, Inc., New York, USA, 1993

8. B.L. Smith "Summary Report for MEGAPIE R\&D Task Group X4: Fluid Dynamics and Structure Mechanics", PSI Bericht Nr.06-0 , ISSN 1019-0643, Switzerland, March 2006 